\title{
Mixed Finite Element Solution for the Natural-Gas Dual-Mechanism Model
}

\author{
Mohamed F. El-Amin ${ }^{1,2}$, Jisheng $\mathrm{Kou}^{3}$, Shuyu $\mathrm{Sun}^{4}$, and Jingfa $\mathrm{Li}^{4}$ \\ 1 College of Engineering, Effat University, Jeddah 21478, KSA \\ 2 Mathematics Department, Faculty of Science, Aswan University, Aswan 81528, \\ Egypt \\ 3 School of Mathematics and Statistics, Hubei Engineering University, Xiaogan \\ 432000, Hubei, China \\ 4 King Abdullah University of Science and Technology (KAUST), Thuwal \\ 23955-6900, KSA \\ momousa@effatuniversity.edu.sa
}

\begin{abstract}
The present work is dedicated to studying the transfer of natural gas in shale formations. The governing model was developed on the basis of the model of dual-porosity dual-permeability (DPDP). The mixed finite element method (MFEM) is employed to solve the governing equations numerically. Numerical example is presented and results discussed such as production cumulative rate, pressure and apparent permeability.
\end{abstract}

Keywords: shale-gas, mixed finite element, porous media, IMPES

\section{Introduction}

Shale-gas resources represent around $30 \%$ of the world resources of the natural gas and it has much promise as a relatively new clean energy since its combustion produces much less $\mathrm{CO}_{2}$. However, the gas production from the shale formation does not obey the conventional ways of gas/oil production from conventional reservoirs. The shale-formations have very-low permeabilities compared to the conventional formations. The production efficiency of the natural gas from shales increases as the efficiency of the fracture network increases.

The model of flow in fractured porous media contains the effect of fluid transfer from matrix blocks to fractures with dual-mechanism such as dualporosity dual-permeability. In the conventional models of fractured formations, Darcy's law is employed to describe the flow. However, Darcy's law does not give a suitable description of the flow in shales because the gas flow in the nanosized pores has slippage on the surface. One of the modeling directions for gas transport in shale formations is to adopt the framework of flow in fractured porous media. Using dual-continua mechanisms, an idealized model was built by Warren and Root [1] to describe flow in fractured media. In this model, the matrix field is divided as uniform cubes separated by fractures. The dualcontinuum models have been developed by several researchers (e.g. Bustin et al. 
[2]; and [4]) to model gas transport in shales. These models incorporated different physics such as inertial, gas slippage, Knudsen diffusion, etc. Recently, El-Amin et al. [5] used the dual-porosity dual- permeability model with geomechanical effect. Ge et al. [6] presented a quantitative evaluation for organic related pores in unconventional reservoir. A comprehensive review has been introduced on gas transport in tight and shale formations was done by Salama et al. [7]. El-Amin and coauthors [8-10] have extended the mathematical model of shale-gas flow and developed some numerical or analytical solutions.

In the current work, we present a mixed finite element method (MFEM) which is a locally conservative method [11] to solve the dual-porosity dualpermeability (DPDP) shale-gas model. We presented some numerical tests to show the efficiency of the numerical scheme.

\section{Modeling and Formulation}

In this section, we develop a DPDP model to simulate the natural gas flow in porous media. The DPDP model consists of two mass balance equations, one for flow in matrix blocks and another for flow in fractures. The flow is assumed to be a single-phase and isothermal, and the gravity effect was neglected. The mass balance equation has the form,

$$
\frac{\partial M}{\partial t}+\nabla \cdot \rho \mathbf{u}=Q
$$

such that $t$ is the time; $M$ is the mass-accumulation; $\rho$ is the gas density; $\mathbf{u}$ is the velocity; $Q$ is the source-term. In the matrix blocks, there exist two mechanisms, namely, free gas and absorbed gas. So, the mass accumulation term may be written as,

$$
M_{f r}=\phi \rho,
$$

However, the mass accumulation term for the adsorption process on the matrix surface is,

$$
M_{a d s}=(1-\phi) q_{a},
$$

such that $q_{a}$ is the volume of the adsorbed-gas on the shale-surface. The adsorption is described by the Langmuir isotherm,

$$
q_{a}=\frac{\rho_{s} M_{w} V_{L} p_{m}}{V_{s t d}\left(P_{L}+p_{m}\right)}
$$

where $V_{s t d}$ and $V_{L}$ are, respectively, mole volume under standard conditions and the Langmuir volume. $\rho_{s}$ is the rock density and $M_{w}$ is the molar weight. $P_{L}$ and $p_{m}$ are, respectively, Langmuir pressure and matrix pressure.

Using Eqs. (2)-(4), we get,

$$
M=\phi \rho+(1-\phi) q_{a},
$$

The mass density can be written as, $\rho_{g, d}=\frac{p_{d} M_{w}}{Z R T}, \quad d=m, f . R, T, m, V$ and $Z$ are the universal gas constant, temperature, mass, volume, and compressibility 
factor, respectively. Thus, $Z=\frac{p V}{R T}$, such that $p$ is the pressure. The PengRobinson equation of state has been used to calculate $Z$,

$$
Z^{3}-(1-B) Z^{2}+\left(A-3 B^{3}-2 B\right) Z-\left(A B-B^{2}-B^{3}\right)=0
$$

where $A=\frac{a_{T} p}{R^{2} T^{2}}, \quad B=\frac{b_{T} p}{R T}$. $a$ and $b$ are coefficients depend on the critical properties, i.e., $a_{T}=0.45724 \frac{R^{2} T_{c}^{2}}{p_{c}}, \quad b_{T}=0.0778 \frac{R T_{c}}{p_{c}} \cdot p_{c}$, is the pressure at the critical point, and $T_{c}$ is the temperature at the critical point.

The slippage effect takes place in the case of gas flow in tight reservoirs, therefore, the permeability developed to its apparent version, thus,

$$
\mathbf{K}_{m, a p p}=K_{m}\left(1+\frac{b_{m}}{p_{m}}\right)
$$

where $K_{m}$ is the intrinsic permeability, $b_{m}$ is the Klinkenberg effect.

From all the above formulations, the DPDP model may be written as,

$$
\begin{gathered}
f_{1}\left(p_{m}\right) \frac{\partial p_{m}}{\partial t}-\nabla \cdot\left[\frac{\rho_{m} K_{m}}{\mu}\left(1+\frac{b_{m}}{p_{m}}\right) \nabla p_{m}\right]=-S\left(p_{m}, p_{f}\right) \\
f_{2} \frac{\partial p_{f}}{\partial t}-\nabla \cdot\left[\frac{\rho_{f} K_{f}}{\mu} \nabla p_{f}\right]=S\left(p_{m}, p_{f}\right)-Q\left(p_{f}\right)
\end{gathered}
$$

where $f_{1}\left(p_{m}\right)=\frac{M_{w} \phi_{m}}{R T}+\frac{M_{w} V_{L} \rho_{s}}{V_{s t d}} \frac{P_{L}\left(1-\phi_{m}\right)}{\left(P_{L}+p_{m}\right)^{2}}$ and $f_{2}=\frac{M_{w} \phi_{f}}{R T}$.

The transfer matrix-fracture term is given by,

$$
S\left(p_{m}, p_{f}\right)=\frac{\sigma \rho K_{m}}{\mu}\left(p_{m}-p_{f}\right)
$$

The Peaceman's model is used to describe the production-source term as [12],

$$
Q\left(p_{f}\right)=\frac{\theta K_{f} \rho\left[\bar{p}_{f}-p_{w f}\right]}{\mu \ln \frac{r e}{r_{w}}}
$$

where $r_{e}=r_{c} \sqrt{(\Delta x)^{2}+(\Delta y)^{2}}$ is the drainage radius, $r_{c}$ is a constant and $r_{w}$ is the well radius. If $\theta=2 \pi$, well will be in field center. If $\theta=\pi / 2$, the well will be in the corner. $\sigma$ is the crossflow coefficient and given by, $\sigma=4\left(\frac{1}{L_{x}^{2}}+\frac{1}{L_{y}^{2}}+\frac{1}{L_{z}^{2}}\right)$. $L_{x}, L_{y}$ and $L_{z}$ are, respectively, fracture spacing of $x, y$ and $z . b_{m}$ and $b_{f}$ are constants [4],

$$
b_{m}=\sqrt{\frac{8 \pi R T}{M_{w}}} \frac{1}{r_{w}}\left(\frac{2}{\alpha}-0.995\right) \mu, \quad b_{f}=\sqrt{\frac{\pi R T \phi_{f}}{M_{w} K_{f}}} \mu
$$

The Knudsen diffusion has the form, $D_{k f}=\sqrt{\frac{\pi R T K_{f} \phi_{f}}{M_{w}}} . K_{f}, \phi_{f}$ and $\mu$ are the fractures permeability, the fractures porosity, and $\mu$ is the gas viscosity. $\alpha$ is a constant. 


\section{$3 \quad$ Mixed Finite Element Method}

Assume that $\Omega_{m} \subset \mathbf{R}^{d}, d \in\{1,2,3\}$ is the matrix polygonal/polyhedral matrix Lipschitz domain; and $\Omega_{f} \subset \mathbf{R}^{d}, d \in\{1,2,3\}$ is the fracture polygonal/polyhedral Lipschitz domain. Also, assume that, $\left.\mathbf{L}^{2}(\Omega) \equiv\left(L^{2}(\Omega)\right)^{d}\right)$, such that $L^{2}(\Omega)$ is the standard space with the boundaries, $\partial \Omega_{m}=\Gamma_{D}^{m} \cup \Gamma_{N}^{m}$ and $\partial \Omega_{f}=\Gamma_{D}^{f} \cup \Gamma_{N}^{f}$. The governing equations can be rewritten as,

$$
\begin{gathered}
f_{1}\left(p_{m}\right) \frac{\partial p_{m}}{\partial t}+\nabla \cdot \mathbf{u}_{m}=-S\left(p_{m}, p_{f}\right) \quad \text { in } \quad \Omega_{m} \times(0, T), \\
\mathbf{D}_{m}\left(p_{m}\right)^{-1} \mathbf{u}_{m}=-\nabla p_{m} \quad \text { in } \quad \Omega_{m} \times(0, T), \\
f_{2} \frac{\partial p_{f}}{\partial t}+\nabla \cdot \mathbf{u}_{f}=S\left(p_{m}, p_{f}\right)-Q\left(p_{f}\right) \quad \text { in } \quad \Omega_{f} \times(0, T), \\
\mathbf{D}_{f}\left(p_{f}\right)^{-1} \mathbf{u}_{f}=-\nabla p_{f} \quad \text { in } \quad \Omega_{f} \times(0, T),
\end{gathered}
$$

In order to avoid discontinuity, both of the functions $\mathbf{D}_{m}\left(p_{m}\right)^{-1}$ and $\mathbf{D}_{f}\left(p_{f}\right)^{-1}$ are moved to the left hand side. Similarly, we can rewrite the initial and boundary conditions of the matrix and fracture domains, as follows,

$$
\begin{gathered}
p_{m}(\cdot, 0)=p_{f}(\cdot, 0)=p_{0} \quad \text { in } \quad \Omega_{m} \cup \Omega_{f}, \quad p_{f}(\cdot, t)=p_{w} \quad \text { on } \quad \Gamma_{D}^{f} \times(0, T), \\
\mathbf{u}_{m} \cdot \mathbf{n}=0 \quad \text { on } \quad \Gamma_{N}^{m} \cup \Gamma_{N}^{f} \times(0, T), \quad \mathbf{u}_{f} \cdot \mathbf{n}=0 \quad \text { on } \quad \Gamma_{N}^{f} \times(0, T),
\end{gathered}
$$

such that, $\mathbf{D}_{m}\left(p_{m}\right)=\frac{\rho_{m} \mathbf{K}_{m}}{\mu}\left(1+\frac{b_{m}}{p_{m}}\right)$, and $\mathbf{D}_{f}\left(p_{f}\right)=\frac{\rho_{f} \mathbf{K}_{f}}{\mu}$.

Now, let us define the two Raviart-Thomas space $\left(\mathrm{RT}_{r}\right)$ subspaces on the partition $\mathcal{T}_{h}: V_{h} \subset H(\Omega ; d i v)$ and $W_{h} \subset L^{2}(\Omega)$ such that $r$-th order $(r \geq 0)$. The MFE weak formulations are:

$$
\begin{gathered}
\left(f_{1}\left(p_{m}^{h}\right) \frac{\partial p_{m}^{h}}{\partial t}, \varphi\right)+\left(\nabla \cdot \mathbf{u}_{m}^{h}, \varphi\right)+\left(\mathcal{S}\left(p_{m}^{h}, p_{f}^{h}\right), \varphi\right)=0 \\
\left(\mathbf{D}_{m}\left(p_{m}^{h}\right)^{-1} \mathbf{u}_{m}^{h}, \omega\right)=\left(p_{m}^{h}, \nabla \cdot \omega\right), \\
\left(f_{2} \frac{\partial p_{f}^{h}}{\partial t}, \varphi\right)+\left(\nabla \cdot \mathbf{u}_{f}^{h}, \varphi\right)-\left(\mathcal{S}\left(p_{m}^{h}, p_{f}^{h}\right), \varphi\right)=-\left(Q\left(p_{f}^{h}\right), \varphi\right), \\
\left(\mathbf{D}_{f}\left(p_{f}^{h}\right)^{-1} \mathbf{u}_{f}^{h}, \omega\right)=\left(p_{f}^{h}, \nabla \cdot \omega\right)-\left\langle P_{w}, \omega\right\rangle_{\Gamma_{f}^{D}},
\end{gathered}
$$

for any $\varphi \in W_{h}$ and $\omega \in V_{h} . p_{m}^{h}, p_{f}^{h} \in W_{h}$ and $u_{m}^{h}, u_{f}^{h} \in V_{h}$. 


\section{Numerical Algorithm}

The MFE approximations with a quadrature rule has been employed to get an explicit flux. The backward Euler method is used to discretize the timederivative with a number of $N_{T}$ time-steps, $\Delta t^{n}=t^{n+1}-t^{n}$, such that $n+1$ is the current time, and $n$ is the previous one, and the total time-interval is $[0, T]$. The discretized MFEM equations are given as,

$$
\begin{gathered}
\left(f_{1}{ }^{h, n} \frac{p_{m}^{h, n+1}-p_{m}^{h, n}}{\Delta t}, \varphi\right)+\left(\nabla \cdot \mathbf{u}_{m}^{h, n+1}, \varphi\right)+\left(\mathcal{S}\left(p_{m}^{h, n+1}, p_{f}^{h, n}\right), \varphi\right)=0 \\
\left(\mathbf{D}_{m}\left(p_{m}^{h, n}\right)^{-1} \mathbf{u}_{m}^{h, n+1}, \omega\right)=\left(p_{m}^{h, n+1}, \nabla \cdot \omega\right) \\
\left(f_{2}{ }^{h, n} \frac{p_{f}^{h, n+1}-p_{f}^{h, n}}{\Delta t}, \varphi\right)+\left(\nabla \cdot \mathbf{u}_{f}^{h, n+1}, \varphi\right)-\left(\mathcal{S}\left(p_{m}^{h, n+1}, p_{f}^{h, n+1}\right), \varphi\right)=-\left(Q_{f}^{h, n+1}, \varphi\right) \\
\left(\mathbf{D}_{f}\left(p_{f}^{h, n}\right)^{-1} \mathbf{u}_{f}^{h, n+1}, \omega\right)=\left(p_{f}^{h, n+1}, \nabla \cdot \omega\right)-\left\langle p_{w}, \omega\right\rangle_{\Gamma_{f}^{D}}
\end{gathered}
$$

Give $p_{m}^{h, n}$ and $p_{f}^{h, n}$, the following scheme are used to find pressures and velocities as,

1. Calculate the thermodynamic variables explicitly.

2. Find $p_{m}^{h, n+1}$ and $\mathbf{u}_{m}^{h, n+1}$ by solving (20)-(21).

3. Find $p_{f}^{h, n+1}$ and $\mathbf{u}_{f}^{h, n+1}$ by solving (22)-(23).

\section{$5 \quad$ Results and Discussions}

Numerical test has been presented to show the efficiency of the current scheme. Table 1 shows the physical parameters of the problem under consideration. A $20 \times 20 \mathrm{~m}$ domain has been used for computations. Fig. 1 shows the distributions of matrix/fracture pressures and velocity. Also, the same figure shows the apparent permeability. From this figure, one notice that both matrix and fractures pressures decrease gradually close to the production well. Also, the apparent permeabilities are reduced. Moreover, It is clear that the apparent permeabilities decrease gradually as it goes farther from the well. It also can be observed that the high the apparent permeability the closer to the production-well. This may be due to the reverse relationship with the pressure. The gas production rate and cumulative production at different values of the slippage factor $b_{m}$ are plotted against the production time in Fig. 2. This figure indicates that the slippage factor has a significant effect on the gas production rate.

\section{Conclussions}

This work presents a mixed finite element method (MFEM) to solve the DPDP model of natural gas transport in shales. Results such as apparent permeability, pressure and production cumulative rate are discussed. In the future work, more realistic scenarios and solution properties of the MFEM scheme will be provided. 
Table 1. physical parameters.

\begin{tabular}{llll}
\hline Parameter & Value & Unit & Description \\
\hline$K_{m}$ & $1.00 \mathrm{E}-04 \mathrm{md}$ & Matrix permeability \\
$K_{f}$ & 10 & $\mathrm{md}$ & Fracture permeability \\
$\phi_{m}$ & 0.05 & - & Matrix porosity \\
$\phi_{f}$ & 0.001 & - & Fracture porosity \\
$\mathrm{R}$ & 8.314 & $\mathrm{~m}^{3} \mathrm{~Pa} / \mathrm{mol} \mathrm{K}$ & Gas constant \\
$\mathrm{T}$ & 373 & $\mathrm{~K}$ & Temperature \\
$\mathrm{Z}$ & 1 & - & Compressibility factor \\
$p_{0}$ & 5 & $\mathrm{MPa}$ & Initial reservoir pressure \\
$p_{w}$ & 3 & $\mathrm{MPa}$ & Bottom hole pressure \\
$M_{w}$ & 0.016 & $\mathrm{~kg} / \mathrm{mol}$ & Molecular weight of $\mathrm{CH}$ \\
$V_{s t d}$ & 0.0224 & $\mathrm{~m} / \mathrm{mol}$ & Standard gas volume \\
$P_{L}$ & 6 & $\mathrm{MPa}$ & Langmuir pressure \\
$V_{L}$ & $2.83 \mathrm{E}-03 \mathrm{~m} / \mathrm{kg}$ & Langmuir volume \\
$\rho_{s}$ & 2550 & $\mathrm{~kg} / \mathrm{m}^{3}$ & Shale rock density \\
$\mu$ & $1.02 \mathrm{E}-05 \mathrm{~Pa} \mathrm{~s}$ & Initial gas viscosity \\
$r_{w}$ & 0.1 & $\mathrm{~m}$ & Wellbore radius \\
$L_{x}, L_{y}, L_{z}$ & 0.2 & $\mathrm{~m}$ & Fracture spacing \\
$\alpha$ & 0.8 & - & Constant \\
\hline
\end{tabular}
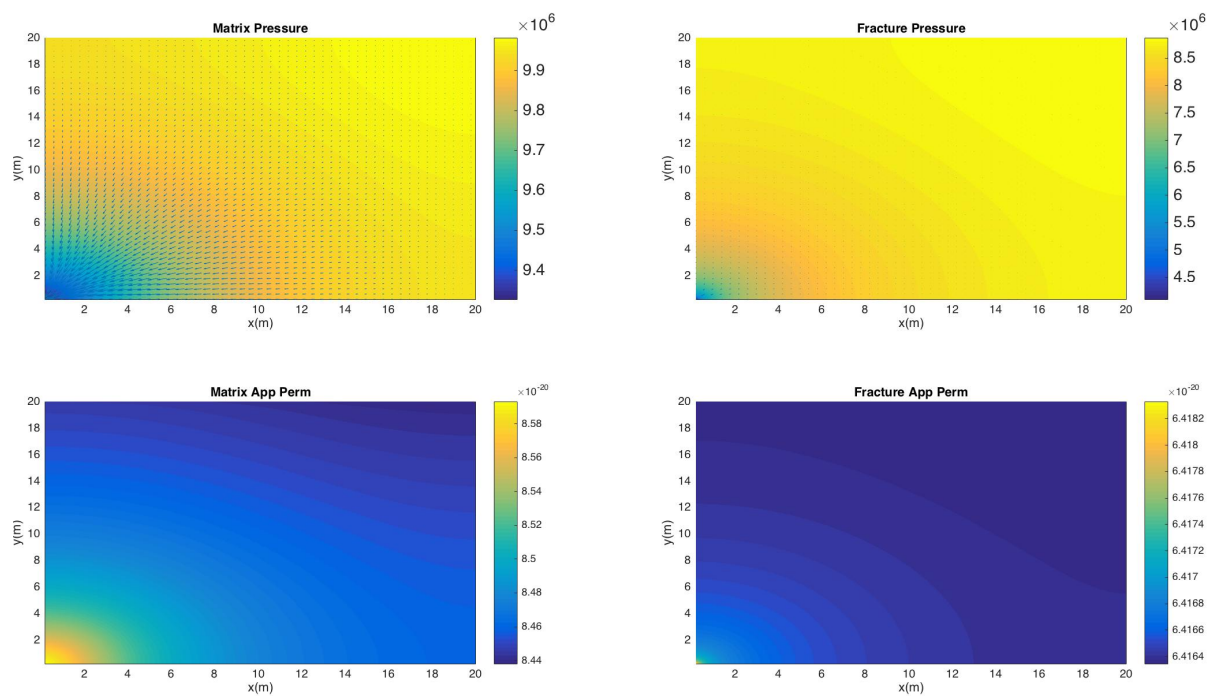

Fig. 1. Distribution of pressure and velocity of matrix and fractures (upper left and right) and apparent permeability of the matrix and fractures (lower left and right). 

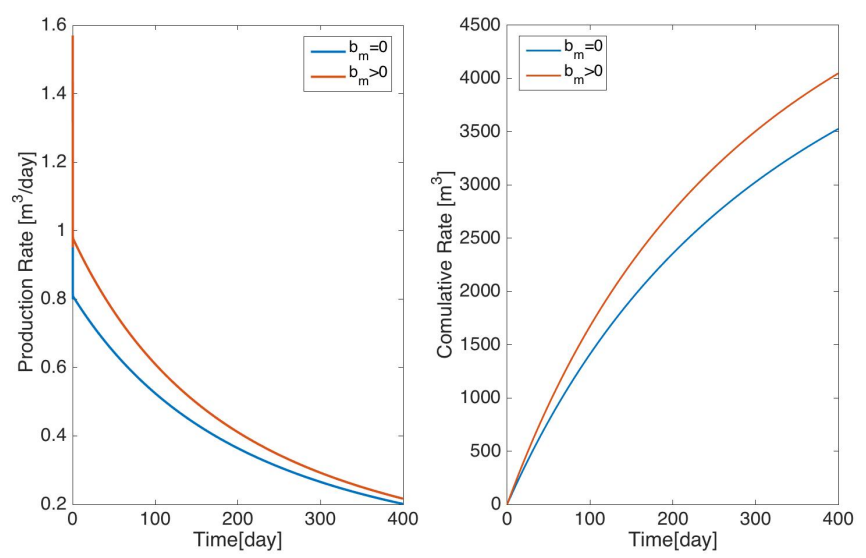

Fig. 2. Gas production rate and cumulative production at different values of $b_{m}$.

\section{References}

1. Warren, J.E., Root, P.J.: The behavior of naturally fractured reservoirs. SPE J. 245-255 (1963).

2. Bustin A, Bustin R, Cui X.: Importance of fabric on the production of gas shales. SPE-114167-MS. In: Unconventional Reservoirs Conference in Colorado, USA, 10-12 Feb. (2008).

3. Kazemi, H.: Pressure transient analysis of naturally fractured reservoirs with uniform fracture distribution. SPE Trans. AIME 246, 451-62 (1969).

4. Javadpour F.: Nanopores and apparent permeability of gas flow in mudrocks (shales and siltstone). J. Canadian Pet. Tech. 48(8): 16-21 (2009).

5. El-Amin, M. F., Kou, J., Sun, S.: Numerical Modeling and Simulation of Shale-Gas Transport with Geomechanical Effect. Trans. Porous Media 126 (3), 779-806 (2019).

6. Ge, X., Fan, Y., Cao, Y., Li, J., Cai, J., Liu, J., Wei, S.: Investigation of organic related pores in unconventional reservoir and its quantitative evaluation. Energy \& Fuels 30(6), 4699-4709 (2016).

7. Salama, A., El-Amin M.F., Kumar, K., Sun, S.: Flow and transport in tight and shale formations. Geofluids, Article ID 4251209 (2017).

8. El-Amin M.F., Amir, S., Salama, A., Urozayev, D., Sun, S.: Comparative study of shale-gas production using single- and dual-continuum approaches. J. Pet. Sc. Eng. 157, 894-905 (2017).

9. El-Amin M.F.: Analytical solution of the apparent-permeability gas-transport equation in porous media. The Euro. Phys. J. Plus 132,129-135 (2017).

10. El-Amin M.F., Radwan, A., Sun, S.: Analytical solution for fractional derivative gas-flow equation in porous media. Results in Physics. 7, 2432-2438 (2017).

11. Brezzi, F., Douglas, J., Marini, L.D.: Two families of mixed finite elements for second order elliptic problems. Numer. Math. 47, 217-235 (1985).

12. Peaceman, D.W.: Interpretation of well-block pressures in numerical reservoir simulation. SPE-6893. In: 52nd Annual Fall Technical Conference and Exhibition, Denver (1977). 